\title{
THE LEFSCHETZ-HOPF THEOREM AND AXIOMS FOR THE LEFSCHETZ NUMBER
}

\author{
MARTIN ARKOWITZ AND ROBERT F. BROWN
}

Received Received 28 August 2003

The reduced Lefschetz number, that is, $L(\cdot)-1$ where $L(\cdot)$ denotes the Lefschetz number, is proved to be the unique integer-valued function $\lambda$ on self-maps of compact polyhedra which is constant on homotopy classes such that (1) $\lambda(f g)=\lambda(g f)$ for $f: X \rightarrow Y$ and $g: Y \rightarrow X$; (2) if $\left(f_{1}, f_{2}, f_{3}\right)$ is a map of a cofiber sequence into itself, then $\lambda\left(f_{1}\right)=$ $\lambda\left(f_{1}\right)+\lambda\left(f_{3}\right) ;(3) \lambda(f)=-\left(\operatorname{deg}\left(p_{1} f e_{1}\right)+\cdots+\operatorname{deg}\left(p_{k} f e_{k}\right)\right)$, where $f$ is a self-map of a wedge of $k$ circles, $e_{r}$ is the inclusion of a circle into the $r$ th summand, and $p_{r}$ is the projection onto the $r$ th summand. If $f: X \rightarrow X$ is a self-map of a polyhedron and $I(f)$ is the fixed-point index of $f$ on all of $X$, then we show that $I(\cdot)-1$ satisfies the above axioms. This gives a new proof of the normalization theorem: if $f: X \rightarrow X$ is a self-map of a polyhedron, then $I(f)$ equals the Lefschetz number $L(f)$ of $f$. This result is equivalent to the Lefschetz-Hopf theorem: if $f: X \rightarrow X$ is a self-map of a finite simplicial complex with a finite number of fixed points, each lying in a maximal simplex, then the Lefschetz number of $f$ is the sum of the indices of all the fixed points of $f$.

\section{Introduction}

Let $X$ be a finite polyhedron and denote by $\tilde{H}_{*}(X)$ its reduced homology with rational coefficients. Then the reduced Euler characteristic of $X$, denoted by $\tilde{\chi}(X)$, is defined by

$$
\tilde{\chi}(X)=\sum_{k}(-1)^{k} \operatorname{dim} \tilde{H}_{k}(X)
$$

Clearly, $\tilde{\chi}(X)$ is just the Euler characteristic minus one. In 1962, Watts [13] characterized the reduced Euler characteristic as follows. Let $\epsilon$ be a function from the set of finite polyhedra with base points to the integers such that (i) $\epsilon\left(S^{0}\right)=1$, where $S^{0}$ is the 0 -sphere, and (ii) $\epsilon(X)=\epsilon(A)+\epsilon(X / A)$, where $A$ is a subpolyhedron of $X$. Then $\epsilon(X)=\tilde{\chi}(X)$.

Let $\mathscr{C}$ be the collection of spaces $X$ of the homotopy type of a finite, connected CWcomplex. If $X \in \mathscr{C}$, we do not assume that $X$ has a base point except when $X$ is a sphere or a wedge of spheres. It is not assumed that maps between spaces with base points are based. A map $f: X \rightarrow X$, where $X \in \mathscr{C}$, induces trivial homomorphisms $f_{* k}: H_{k}(X) \rightarrow H_{k}(X)$ 
of rational homology vector spaces for all $j>\operatorname{dim} X$. The Lefschetz number $L(f)$ of $f$ is defined by

$$
L(f)=\sum_{k}(-1)^{k} \operatorname{Tr} f_{* k}
$$

where $\operatorname{Tr}$ denotes the trace. The reduced Lefschetz number $\tilde{L}$ is given by $\tilde{L}(f)=L(f)-1$ or, equivalently, by considering the rational, reduced homology homomorphism induced by $f$.

Since $\tilde{L}(\mathrm{id})=\tilde{\chi}(X)$, where id : $X \rightarrow X$ is the identity map, Watts's Theorem suggests an axiomatization for the reduced Lefschetz number which we state below in Theorem 1.1.

For $k \geq 1$, denote by $\bigvee^{k} S^{n}$ the wedge of $k$ copies of the $n$-sphere $S^{n}, n \geq 1$. If we write $\vee^{k} S^{n}$ as $S_{1}^{n} \vee S_{2}^{n} \vee \cdots \vee S_{k}^{n}$, where $S_{j}^{n}=S^{n}$, then we have inclusions $e_{j}: S_{j}^{n} \rightarrow \vee^{k} S^{n}$ into the $j$ th summand and projections $p_{j}: \bigvee^{k} S^{n} \rightarrow S_{j}^{n}$ onto the $j$ th summand, for $j=1, \ldots, k$. If $f: \bigvee^{k} S^{n} \rightarrow \bigvee^{k} S^{n}$ is a map, then $f_{j}: S_{j}^{n} \rightarrow S_{j}^{n}$ denotes the composition $p_{j} f e_{j}$. The degree of a map $f: S^{n} \rightarrow S^{n}$ is denoted by $\operatorname{deg}(f)$.

We characterize the reduced Lefschetz number as follows.

Theorem 1.1. The reduced Lefschetz number $\tilde{L}$ is the unique function $\lambda$ from the set of self-maps of spaces in $\mathscr{C}$ to the integers that satisfies the following conditions.

(1) (Homotopy axiom) If $f, g: X \rightarrow X$ are homotopic maps, then $\lambda(f)=\lambda(g)$.

(2) (Cofibration axiom) If $A$ is a subpolyhedron of $X, A \rightarrow X \rightarrow X / A$ is the resulting cofiber sequence, and there exists a commutative diagram

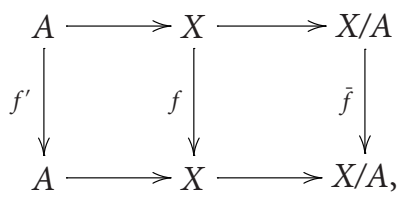

then $\lambda(f)=\lambda\left(f^{\prime}\right)+\lambda(\bar{f})$.

(3) (Commutativity axiom) If $f: X \rightarrow Y$ and $g: Y \rightarrow X$ are maps, then $\lambda(g f)=\lambda(f g)$.

(4) (Wedge of circles axiom) If $f: \bigvee^{k} S^{1} \rightarrow \bigvee^{k} S^{1}$ is a map, $k \geq 1$, then

$$
\lambda(f)=-\left(\operatorname{deg}\left(f_{1}\right)+\cdots+\operatorname{deg}\left(f_{k}\right)\right),
$$

where $f_{j}=p_{j} f e_{j}$.

In an unpublished dissertation [10], Hoang extended Watts's axioms to characterize the reduced Lefschetz number for basepoint-preserving self-maps of finite polyhedra. His list of axioms is different from, but similar to, those in Theorem 1.1.

One of the classical results of fixed-point theory is the following theorem.

Theorem 1.2 (Lefschetz-Hopf). If $f: X \rightarrow X$ is a map of a finite polyhedron with a finite set of fixed points, each of which lies in a maximal simplex of $X$, then $L(f)$ is the sum of the indices of all the fixed points of $f$. 
The history of this result is described in [3], see also [8, page 458]. A proof that depends on a delicate argument due to Dold [4] can be found in [2] and, in a more condensed form, in [5]. In an appendix to his dissertation [12], McCord outlined a possibly more direct argument, but no details were published. The book of Granas and Dugundji [8, pages 441-450] presents an argument based on classical techniques of Hopf [11]. We use the characterization of the reduced Lefschetz number in Theorem 1.1 to prove the Lefschetz-Hopf theorem in a quite natural manner by showing that the fixed-point index satisfies the axioms of Theorem 1.1. That is, we prove the following theorem.

Theorem 1.3 (normalization property). If $f: X \rightarrow X$ is any map of a finite polyhedron, then $L(f)=i(X, f, X)$, the fixed-point index of $f$ on all of $X$.

The Lefschetz-Hopf theorem follows from the normalization property by the additivity property of the fixed-point index. In fact, these two statements are equivalent. The Hopf construction [2, page 117] implies that a map $f$ from a finite polyhedron to itself is homotopic to a map that satisfies the hypotheses of the Lefschetz-Hopf theorem. Thus, the homotopy and additivity properties of the fixed-point index imply that the normalization property follows from the Lefschetz-Hopf theorem.

\section{Lefschetz numbers and exact sequences}

In this section, all vector spaces are over a fixed field $F$, which will not be mentioned, and are finite dimensional. A graded vector space $V=\left\{V_{n}\right\}$ will always have the following properties: (1) each $V_{n}$ is finite dimensional and (2) $V_{n}=0$, for $n<0$ and for $n>N$, for some nonnegative integer $N$. A map $f: V \rightarrow W$ of graded vector spaces $V=\left\{V_{n}\right\}$ and $W=\left\{W_{n}\right\}$ is a sequence of linear transformations $f_{n}: V_{n} \rightarrow W_{n}$. For a map $f: V \rightarrow V$, the Lefschetz number is defined by

$$
L(f)=\sum_{n}(-1)^{n} \operatorname{Tr} f_{n}
$$

The proof of the following lemma is straightforward, and hence omitted.

LEMma 2.1. Given a map of short exact sequences of vector spaces

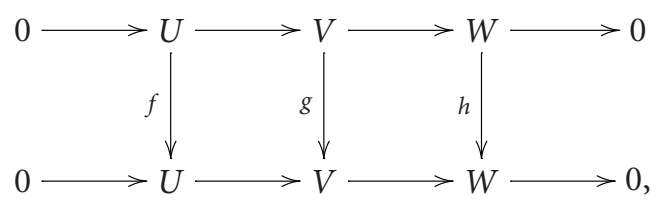

then $\operatorname{Tr} g=\operatorname{Tr} f+\operatorname{Tr} h$.

Theorem 2.2. Let $A, B$, and $C$ be graded vector spaces with maps $\alpha: A \rightarrow B, \beta: B \rightarrow C$ and self-maps $f: A \rightarrow A, g: B \rightarrow B$, and $h: C \rightarrow C$. If, for every $n$, there is a linear transformation 
4 Lefschetz number

$\partial_{n}: C_{n} \rightarrow A_{n-1}$ such that the following diagram is commutative and has exact rows:

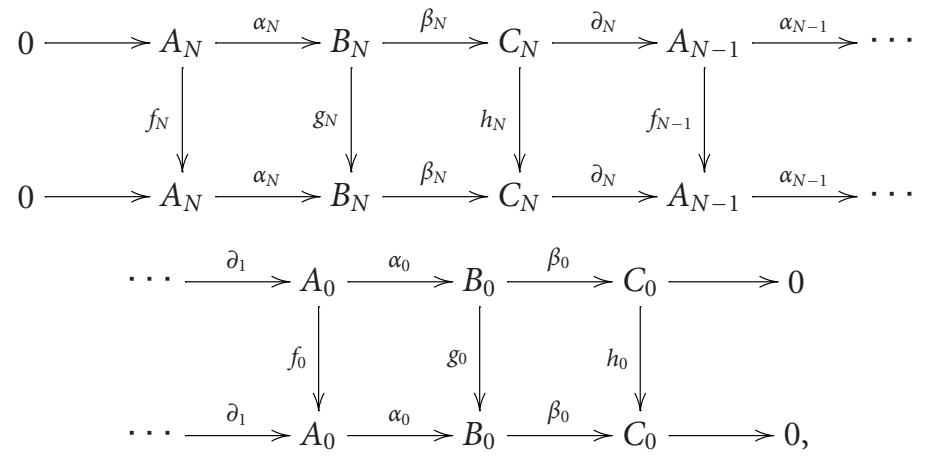

then

$$
L(g)=L(f)+L(h)
$$

Proof. Let Im denote the image of a linear transformation and consider the commutative diagram

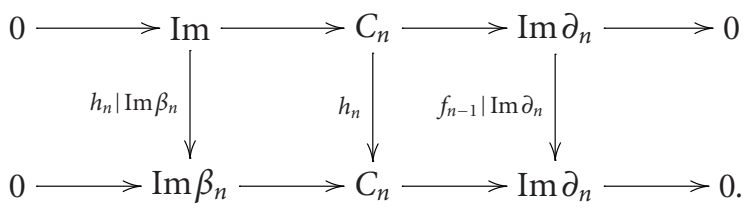

By Lemma 2.1, $\operatorname{Tr}\left(h_{n}\right)=\operatorname{Tr}\left(h_{n} \mid \operatorname{Im} \beta_{n}\right)+\operatorname{Tr}\left(f_{n-1} \mid \operatorname{Im} \partial_{n}\right)$. Similarly, the commutative diagram

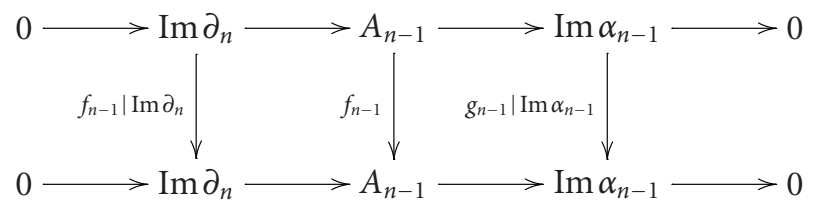

yields $\operatorname{Tr}\left(f_{n-1} \mid \operatorname{Im} \partial_{n}\right)=\operatorname{Tr}\left(f_{n-1}\right)-\operatorname{Tr}\left(g_{n-1} \mid \operatorname{Im} \alpha_{n-1}\right)$. Therefore,

$$
\operatorname{Tr}\left(h_{n}\right)=\operatorname{Tr}\left(h_{n} \mid \operatorname{Im} \beta_{n}\right)+\operatorname{Tr}\left(f_{n-1}\right)-\operatorname{Tr}\left(g_{n-1} \mid \operatorname{Im} \alpha_{n-1}\right) .
$$

Now consider

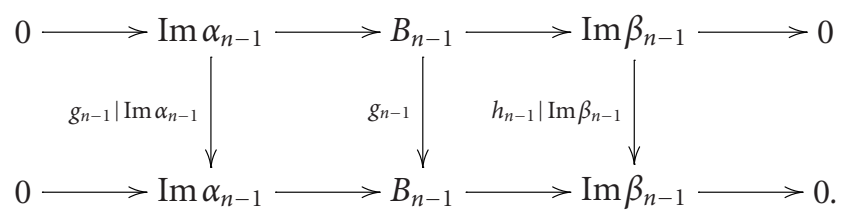


So $\operatorname{Tr}\left(g_{n-1} \mid \operatorname{Im} \alpha_{n-1}\right)=\operatorname{Tr}\left(g_{n-1}\right)-\operatorname{Tr}\left(h_{n-1} \mid \operatorname{Im} \beta_{n-1}\right)$. Putting this all together, we obtain

$$
\operatorname{Tr}\left(h_{n}\right)=\operatorname{Tr}\left(h_{n} \mid \operatorname{Im} \beta_{n}\right)+\operatorname{Tr}\left(f_{n-1}\right)-\operatorname{Tr}\left(g_{n-1}\right)+\operatorname{Tr}\left(h_{n-1} \mid \operatorname{Im} \beta_{n-1}\right) .
$$

We next look at the left end of diagram (2.3) and get

$$
0=\operatorname{Tr}\left(h_{N+1}\right)=\operatorname{Tr}\left(f_{N}\right)-\operatorname{Tr}\left(g_{N}\right)+\operatorname{Tr}\left(h_{N} \mid \operatorname{Im} \beta_{N}\right)
$$

and at the right end which gives

$$
\operatorname{Tr}\left(h_{1}\right)=\operatorname{Tr}\left(h_{1} \mid \operatorname{Im} \beta_{1}\right)+\operatorname{Tr}\left(f_{0}\right)-\operatorname{Tr}\left(g_{0}\right)+\operatorname{Tr}\left(h_{0}\right)
$$

A simple calculation now yields (where a homomorphism with a negative subscript is the zero homomorphism)

$$
\begin{aligned}
& \sum_{n=0}^{N}(-1)^{n} \operatorname{Tr}\left(h_{n}\right) \\
& \quad=\sum_{n=0}^{N+1}(-1)^{n}\left(\operatorname{Tr}\left(h_{n} \mid \operatorname{Im} \beta_{n}\right)+\operatorname{Tr}\left(f_{n-1}\right)-\operatorname{Tr}\left(g_{n-1}\right)+\operatorname{Tr}\left(h_{n-1} \mid \operatorname{Im} \beta_{n-1}\right)\right) \\
& \quad=-\sum_{n=0}^{N}(-1)^{n} \operatorname{Tr}\left(f_{n}\right)+\sum_{n=0}^{N}(-1)^{n} \operatorname{Tr}\left(g_{n}\right) .
\end{aligned}
$$

Therefore, $L(h)=-L(f)+L(g)$.

A more condensed version of this argument has recently been published, see [8, page 420].

We next give some simple consequences of Theorem 2.2.

If $f:(X, A) \rightarrow(X, A)$ is a self-map of a pair, where $X, A \in \mathscr{C}$, then $f$ determines $f_{X}$ : $X \rightarrow X$ and $f_{A}: A \rightarrow A$. The map $f$ induces homomorphisms $f_{* k}: H_{k}(X, A) \rightarrow H_{k}(X, A)$ of relative homology with coefficients in $F$. The relative Lefschetz number $L(f ; X, A)$ is defined by

$$
L(f ; X, A)=\sum_{k}(-1)^{k} \operatorname{Tr} f_{* k}
$$

Applying Theorem 2.2 to the homology exact sequence of the pair $(X, A)$, we obtain the following corollary.

Corollary 2.3. If $f:(X, A) \rightarrow(X, A)$ is a map of pairs, where $X, A \in \mathscr{C}$, then

$$
L(f ; X, A)=L\left(f_{X}\right)-L\left(f_{A}\right)
$$

This result was obtained by Bowszyc [1]. 
Corollary 2.4. Suppose $X=P \cup Q$, where $X, P, Q \in \mathscr{C}$ and $(X ; P, Q)$ is a proper triad [6, page 34]. If $f: X \rightarrow X$ is a map such that $f(P) \subseteq P$ and $f(Q) \subseteq Q$, then, for $f_{P}, f_{Q}$, and $f_{P \cap Q}$ being the restrictions of $f$ to $P, Q$, and $P \cap Q$, respectively, there exists

$$
L(f)=L\left(f_{P}\right)+L\left(f_{Q}\right)-L\left(f_{P \cap Q}\right)
$$

Proof. The map $f$ and its restrictions induce a map of the Mayer-Vietoris homology sequence [6, page 39] to itself, so the result follows from Theorem 2.2.

A similar result was obtained by Ferrario [7, Theorem 3.2.1].

Our final consequence of Theorem 2.2 will be used in the characterization of the reduced Lefschetz number.

Corollary 2.5. If $A$ is a subpolyhedron of $X, A \rightarrow X \rightarrow X / A$ is the resulting cofiber sequence of spaces in $\mathscr{C}$ and there exists a commutative diagram

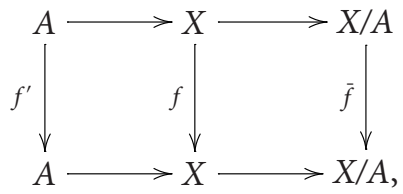

then

$$
L(f)=L\left(f^{\prime}\right)+L(\bar{f})-1
$$

Proof. We apply Theorem 2.2 to the homology cofiber sequence. The "minus one" on the right-hand side arises because such sequence ends with

$$
\longrightarrow H_{0}(A) \longrightarrow H_{0}(X) \longrightarrow \tilde{H}_{0}(X / A) \longrightarrow 0
$$

\section{Characterization of the Lefschetz number}

Throughout this section, all spaces are assumed to lie in $\mathscr{C}$.

We let $\lambda$ be a function from the set of self-maps of spaces in $\mathscr{C}$ to the integers that satisfies the homotopy axiom, cofibration axiom, commutativity axiom, and wedge of circles axiom of Theorem 1.1 as stated in the introduction.

We draw a few simple consequences of these axioms. From the commutativity and homotopy axioms, we obtain the following lemma.

Lemma 3.1. If $f: X \rightarrow X$ is a map and $h: X \rightarrow Y$ is a homotopy equivalence with homotopy inverse $k: Y \rightarrow X$, then $\lambda(f)=\lambda(h f k)$.

Lemma 3.2. If $f: X \rightarrow X$ is homotopic to a constant map, then $\lambda(f)=0$. 
Proof. Let $*$ be a one-point space and $*: * \rightarrow *$ the unique map. From the map of cofiber sequences

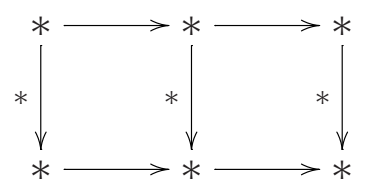

and the cofibration axiom, we have $\lambda(*)=\lambda(*)+\lambda(*)$, and therefore $\lambda(*)=0$. Write any constant map $c: X \rightarrow X$ as $c(x)=*$, for some $* \in X$, let $e: * \rightarrow X$ be inclusion and $p: X \rightarrow *$ projection. Then $c=e p$ and $p e=*$, and so $\lambda(c)=0$ by the commutativity axiom. The lemma follows from the homotopy axiom.

If $X$ is a based space with base point $*$, that is, a sphere or wedge of spheres, then the cone and suspension of $X$ are defined by $C X=X \times I /(X \times 1 \cup * \times I)$ and $\Sigma X=C X /(X \times$ $0)$, respectively.

Lemma 3.3. If $X$ is a based space, $f: X \rightarrow X$ is a based map, and $\Sigma f: \Sigma X \rightarrow \Sigma X$ is the suspension of $f$, then $\lambda(\Sigma f)=-\lambda(f)$.

Proof. Consider the maps of cofiber sequences

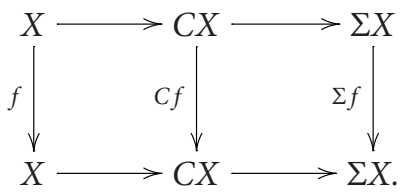

Since $C X$ is contractible, $C f$ is homotopic to a constant map. Therefore, by Lemma 3.2 and the cofibration axiom,

$$
0=\lambda(C f)=\lambda(\Sigma f)+\lambda(f)
$$

LEMMA 3.4. For any $k \geq 1$ and $n \geq 1$, if $f: \bigvee^{k} S^{n} \rightarrow \bigvee^{k} S^{n}$ is a map, then

$$
\lambda(f)=(-1)^{n}\left(\operatorname{deg}\left(f_{1}\right)+\cdots+\operatorname{deg}\left(f_{k}\right)\right),
$$

where $e_{j}: S^{n} \rightarrow \bigvee^{k} S^{n}$ and $p_{j}: \bigvee^{k} S^{n} \rightarrow S^{n}$, for $j=1, \ldots, k$, are the inclusions and projections, respectively, and $f_{j}=p_{j} f e_{j}$.

Proof. The proof is by induction on the dimension $n$ of the spheres. The case $n=1$ is the wedge of circles axiom. If $n \geq 2$, then the map $f: \bigvee^{k} S^{n} \rightarrow \bigvee^{k} S^{n}$ is homotopic to a based map $f^{\prime}: \bigvee^{k} S^{n} \rightarrow \bigvee^{k} S^{n}$. Then $f^{\prime}$ is homotopic to $\Sigma g$, for some map $g: \bigvee^{k} S^{n-1} \rightarrow$ $\bigvee^{k} S^{n-1}$. Note that if $g_{j}: S_{j}^{n-1} \rightarrow S_{j}^{n-1}$, then $\Sigma g_{j}$ is homotopic to $f_{j}: S_{j}^{n} \rightarrow S_{j}^{n}$. Therefore, by 
Lemma 3.3 and the induction hypothesis,

$$
\begin{aligned}
\lambda(f) & =\lambda\left(f^{\prime}\right)=-\lambda(g)=-(-1)^{n-1}\left(\operatorname{deg}\left(g_{1}\right)+\cdots+\operatorname{deg}\left(g_{k}\right)\right) \\
& =(-1)^{n}\left(\operatorname{deg}\left(f_{1}\right)+\cdots+\operatorname{deg}\left(f_{k}\right)\right) .
\end{aligned}
$$

Proof of Theorem 1.1. Since $\tilde{L}(f)=L(f)-1$, Corollary 2.5 implies that $\tilde{L}$ satisfies the cofibration axiom. We next show that $\tilde{L}$ satisfies the wedge of circles axiom. There is an isomorphism $\theta: \bigoplus^{k} H_{1}\left(S^{1}\right) \rightarrow H_{1}\left(\bigvee^{k} S^{1}\right)$ defined by $\theta\left(x_{1}, \ldots, x_{k}\right)=e_{1 *}\left(x_{1}\right)+\cdots+e_{k *}\left(x_{k}\right)$, where $x_{i} \in H_{1}\left(S^{1}\right)$. The inverse $\theta^{-1}: H_{1}\left(\bigvee^{k} S^{1}\right) \rightarrow \bigoplus^{k} H_{1}\left(S^{1}\right)$ is given by $\theta^{-1}(y)=$ $\left(p_{1 *}(y), \ldots, p_{k *}(y)\right)$. If $u \in H_{1}\left(S^{1}\right)$ is a generator, then a basis for $H_{1}\left(\bigvee^{k} S^{1}\right)$ is $e_{1 *}(u), \ldots$, $e_{k *}(u)$. By calculating the trace of $f_{* 1}: H_{1}\left(\bigvee^{k} S^{1}\right) \rightarrow H_{1}\left(\bigvee^{k} S^{1}\right)$ with respect to this basis, we obtain $\tilde{L}(f)=-\left(\operatorname{deg}\left(f_{1}\right)+\cdots+\operatorname{deg}\left(f_{k}\right)\right)$. The remaining axioms are obviously satisfied by $\tilde{L}$. Thus $\tilde{L}$ satisfies the axioms of Theorem 1.1 .

Now suppose $\lambda$ is a function from the self-maps of spaces in $\mathscr{C}$ to the integers that satisfies the axioms. We regard $X$ as a connected, finite $C W$-complex and proceed by induction on the dimension of $X$. If $X$ is 1-dimensional, then it is the homotopy type of a wedge of circles. By Lemma 3.1, we can regard $f$ as a self-map of $\bigvee^{k} S^{1}$, and so the wedge of circles axiom gives

$$
\lambda(f)=-\left(\operatorname{deg}\left(f_{1}\right)+\cdots+\operatorname{deg}\left(f_{k}\right)\right)=\tilde{L}(f) .
$$

Now suppose that $X$ is $n$-dimensional and let $X^{n-1}$ denote the $(n-1)$-skeleton of $X$. Then $f$ is homotopic to a cellular map $g: X \rightarrow X$ by the cellular approximation theorem [9, Theorem 4.8, page 349]. Thus $g\left(X^{n-1}\right) \subseteq X^{n-1}$, and so we have a commutative diagram

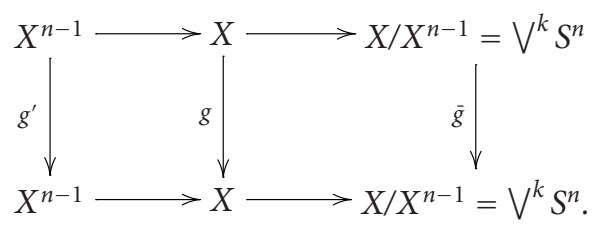

Then, by the cofibration axiom, $\lambda(g)=\lambda\left(g^{\prime}\right)+\lambda(\bar{g})$. Lemma 3.4 implies that $\lambda(\bar{g})=\tilde{L}(\bar{g})$. So, applying the induction hypothesis to $g^{\prime}$, we have $\lambda(g)=\tilde{L}\left(g^{\prime}\right)+\tilde{L}(\bar{g})$. Since we have seen that the reduced Lefschetz number satisfies the cofibration axiom, we conclude that $\lambda(g)=\tilde{L}(g)$. By the homotopy axiom, $\lambda(f)=\tilde{L}(f)$.

\section{The normalization property}

Let $X$ be a finite polyhedron and $f: X \rightarrow X$ a map. Denote by $I(f)$ the fixed-point index of $f$ on all of $X$, that is, $I(f)=i(X, f, X)$ in the notation of [2] and let $\tilde{I}(f)=I(f)-1$.

In this section, we prove Theorem 1.3 by showing that, with rational coefficients, $I(f)=L(f)$.

Proof of Theorem 1.3. We will prove that $\tilde{I}$ satisfies the axioms, and therefore, by Theorem 1.1, $\tilde{I}(f)=\tilde{L}(f)$. The homotopy and commutativity axioms are well-known properties of the fixed-point index (see [2, pages 59-62]). 
To show that $\tilde{I}$ satisfies the cofibration axiom, it suffices to consider $A$ a subpolyhedron of $X$ and $f(A) \subseteq A$. Let $f^{\prime}: A \rightarrow A$ denote the restriction of $f$ and $\bar{f}: X / A \rightarrow X / A$ the map induced on quotient spaces. Let $r: U \rightarrow A$ be a deformation retraction of a neighborhood of $A$ in $X$ onto $A$ and let $L$ be a subpolyhedron of a barycentric subdivision of $X$ such that $A \subseteq \operatorname{int} L \subseteq L \subseteq U$. By the homotopy extension theorem, there is a homotopy $H: X \times I \rightarrow$ $X$ such that $H(x, 0)=f(x)$ for all $x \in X, H(a, t)=f(a)$ for all $a \in A$, and $H(x, 1)=f r(x)$ for all $x \in L$. If we set $g(x)=H(x, 1)$, then, since there are no fixed points of $g$ on $L-A$, the additivity property implies that

$$
I(g)=i(X, g, \operatorname{int} L)+i(X, g, X-L) .
$$

We discuss each summand of (4.1) separately. We begin with $i(X, g$, int $L)$. Since $g(L) \subseteq$ $A \subseteq L$, it follows from the definition of the index (see [2, page 56]) that $i(X, g$, int $L)=$ $i(L, g$, int $L)$. Moreover, $i(L, g$, int $L)=i(L, g, L)$ since there are no fixed points on $L-\operatorname{int} L$ (the excision property of the index). Let $e: A \rightarrow L$ be inclusion, then, by the commutativity property [2, page 62], we have

$$
i(L, g, L)=i(L, e g, L)=i(A, g e, A)=I\left(f^{\prime}\right)
$$

because $f(a)=g(a)$ for all $a \in A$.

Next we consider the summand $i(X, g, X-L)$ of (4.1). Let $\pi: X \rightarrow X / A$ be the quotient map, set $\pi(A)=*$, and note that $\pi^{-1}(*)=A$. If $\bar{g}: X / A \rightarrow X / A$ is induced by $g$, the restriction of $\bar{g}$ to the neighborhood $\pi(\operatorname{int} L)$ of $*$ in $X / A$ is constant, so $i(X / A, \bar{g}, \pi(\operatorname{int} L))=$ 1. If we denote the set of fixed points of $\bar{g}$ with $*$ deleted by $\mathrm{Fix}_{*} \bar{g}$, then $\mathrm{Fix}_{*} \bar{g}$ is in the open subset $X / A-\pi(L)$ of $X / A$. Let $W$ be an open subset of $X / A$ such that $\mathrm{Fix}_{*} \bar{g} \subseteq W \subseteq$ $X / A-\pi(L)$ with the property $\bar{g}(W) \cap \pi(L)=\varnothing$. By the additivity property, we have

$$
I(\bar{g})=i(X / A, \bar{g}, \pi(\operatorname{int} L))+i(X / A, \bar{g}, W)=1+i(X / A, \bar{g}, W) .
$$

Now, identifying $X-L$ with the corresponding subset $\pi(X-L)$ of $X / A$ and identifying the restrictions of $\bar{g}$ and $g$ to those subsets, we have $i(X / A, \bar{g}, W)=i\left(X, g, \pi^{-1}(W)\right)$. The excision property of the index implies that $i\left(X, g, \pi^{-1}(W)\right)=i(X, g, X-L)$. Thus we have determined the second summand of (4.1): $i(X, g, X-L)=I(\bar{g})-1$.

Therefore, from (4.1) we obtain $I(g)=I\left(f^{\prime}\right)+I(\bar{g})-1$. The homotopy property then tells us that

$$
I(f)=I\left(f^{\prime}\right)+I(\bar{f})-1
$$

since $f$ is homotopic to $g$ and $\bar{f}$ is homotopic to $\bar{g}$. We conclude that $\tilde{I}$ satisfies the cofibration axiom.

It remains to verify the wedge of circles axiom. Let $X=\vee^{k} S^{1}=S_{1}^{1} \vee \cdots \vee S_{k}^{1}$ be a wedge of circles with basepoint $*$ and $f: X \rightarrow X$ a map. We first verify the axiom in the case $k=1$. We have $f: S^{1} \rightarrow S^{1}$ and we denote its degree by $\operatorname{deg}(f)=d$. We regard $S^{1} \subseteq \mathbb{C}$, the complex numbers. Then $f$ is homotopic to $g_{d}$, where $g_{d}(z)=z^{d}$ has $|d-1|$ fixed points for $d \neq 1$. The fixed-point index of $g_{d}$ in a neighborhood of a fixed point that contains no other fixed point of $g_{d}$ is -1 if $d \geq 2$ and is 1 if $d \leq 0$. Since $g_{1}$ is homotopic to 
a map without fixed points, we see that $I\left(g_{d}\right)=-d+1$ for all integers $d$. We have shown that $I(f)=-\operatorname{deg}(f)+1$.

Now suppose $k \geq 2$. If $f(*)=*$, then, by the homotopy extension theorem, $f$ is homotopic to a map which does not fix $*$. Thus we may assume, without loss of generality, that $f(*) \in S_{1}^{1}-\{*\}$. Let $V$ be a neighborhood of $f(*)$ in $S_{1}^{1}-\{*\}$ such that there exists a neighborhood $U$ of $*$ in $X$, disjoint from $V$, with $f(\bar{U}) \subseteq V$. Since $\bar{U}$ contains no fixed point of $f$ and the open subsets $S_{j}^{1}-\bar{U}$ of $X$ are disjoint, the additivity property implies

$$
I(f)=i\left(X, f, S_{1}^{1}-\bar{U}\right)+\sum_{j=2}^{k} i\left(X, f, S_{j}^{1}-\bar{U}\right) .
$$

The additivity property also implies that

$$
I\left(f_{j}\right)=i\left(S_{j}^{1}, f_{j}, S_{j}^{1}-\bar{U}\right)+i\left(S_{j}^{1}, f_{j}, S_{j}^{1} \cap U\right) .
$$

There is a neighborhood $W_{j}$ of $(\operatorname{Fix} f) \cap S_{j}^{1}$ in $S_{j}^{1}$ such that $f\left(\bar{W}_{j}\right) \subseteq S_{j}^{1}$. Thus $f_{j}(x)=f(x)$ for $x \in W_{j}$, and therefore, by the excision property,

$$
i\left(S_{j}^{1}, f_{j}, S_{j}^{1}-\bar{U}\right)=i\left(S_{j}^{1}, f_{j}, W_{j}\right)=i\left(X, f, W_{j}\right)=i\left(X, f, S_{j}^{1}-\bar{U}\right) .
$$

Since $f(\bar{U}) \subseteq S_{1}^{1}$, then $f_{1}(x)=f(x)$ for all $x \in \bar{U} \cap S_{1}^{1}$. There are no fixed points of $f$ in $\bar{U}$, so $i\left(S_{1}^{1}, f_{1}, S_{1}^{1} \cap U\right)=0$, and thus, $I\left(f_{1}\right)=i\left(X, f, S_{1}^{1}-\bar{U}\right)$ by (4.6) and (4.7).

For $j \geq 2$, the fact that $f_{j}(U)=*$ gives us $i\left(S_{j}^{1}, f_{j}, S_{j}^{1} \cap U\right)=1$, so $I\left(f_{j}\right)=i\left(X, f, S_{j}^{1}-\right.$ $\bar{U})+1$ by (4.6) and (4.7). Since $f_{j}: S_{j}^{1} \rightarrow S_{j}^{1}$, the $k=1$ case of the argument tells us that $I\left(f_{j}\right)=-\operatorname{deg}\left(f_{j}\right)+1$ for $j=1,2, \ldots, k$. In particular, $i\left(X, f, S_{1}^{1}-\bar{U}\right)=-\operatorname{deg}\left(f_{1}\right)+1$, whereas, for $j \geq 2$, we have $i\left(X, f, S_{j}^{1}-\bar{U}\right)=-\operatorname{deg}\left(f_{j}\right)$. Therefore, by (4.5),

$$
I(f)=i\left(X, f, S_{1}^{1}-\bar{U}\right)+\sum_{j=2}^{k} i\left(X, f, S_{j}^{1}-\bar{U}\right)=-\sum_{j=1}^{k} \operatorname{deg}\left(f_{j}\right)+1 .
$$

This completes the proof of Theorem 1.3.

\section{Acknowledgment}

We thank Jack Girolo for carefully reading a draft of this paper and giving us helpful suggestions.

\section{References}

[1] C. Bowszyc, Fixed point theorems for the pairs of spaces, Bull. Acad. Polon. Sci. Sér. Sci. Math. Astronom. Phys. 16 (1968), 845-850.

[2] R. F. Brown, The Lefschetz Fixed Point Theorem, Scott Foresman, London, 1971.

[3] _ Fixed point theory, History of Topology, North-Holland, Amsterdam, 1999, pp. 271299.

[4] A. Dold, Fixed point index and fixed point theorem for Euclidean neighborhood retracts, Topology 4 (1965), 1-8. 
[5] Lectures on Algebraic Topology, 2nd ed., Grundlehren der Mathematischen Wissenschaften, vol. 200, Springer-Verlag, Berlin, 1980.

[6] S. Eilenberg and N. Steenrod, Foundations of Algebraic Topology, Princeton University Press, New Jersey, 1952.

[7] D. Ferrario, Generalized Lefschetz numbers of pushout maps, Topology Appl. 68 (1996), no. 1, $67-81$.

[8] A. Granas and J. Dugundji, Fixed Point Theory, Springer-Verlag, New York, 2003.

[9] A. Hatcher, Algebraic Topology, Cambridge University Press, Cambridge, 2002.

[10] B. Hoang, Classical and generalized Lefschetz numbers, Ph.D. thesis, UCLA, California, 1985.

[11] H. Hopf, Über die algebraische Anzahl von Fixpunkten, Math. Z. 29 (1929), 493-524 (German).

[12] D. McCord, The converse of the Lefschetz fixed point theorem for surfaces and higher dimensional manifolds, Ph.D. thesis, University of Wisconsin, Wisconsin, 1970.

[13] C. E. Watts, On the Euler characteristic of polyhedra, Proc. Amer. Math. Soc. 13 (1962), 304306.

Martin Arkowitz: Department of Mathematics, Dartmouth College, Hanover, NH 03755-1890, USA

E-mail address: martin.a.arkowitz@dartmouth.edu

Robert F. Brown: Department of Mathematics, University of California, Los Angeles, CA 900951555 , USA

E-mail address: rfb@math.ucla.edu 